Internat. J. Math. \& Math. Sci.

Vol. 23, No. 5 (2000) 335-342

S0161171200001848

(C) Hindawi Publishing Corp.

\title{
MEAN NUMBER OF REAL ZEROS OF A RANDOM HYPERBOLIC POLYNOMIAL
}

\author{
J. ERNEST WILKINS, JR.
}

(Received 25 March 1998)

\begin{abstract}
Consider the random hyperbolic polynomial, $f(x)=1^{p} a_{1} \cosh x+\cdots+n^{p} \times$ $a_{n} \cosh n x$, in which $n$ and $p$ are integers such that $n \geq 2, p \geq 0$, and the coefficients $a_{k}(k=1,2, \ldots, n)$ are independent, standard normally distributed random variables. If $v_{n p}$ is the mean number of real zeros of $f(x)$, then we prove that $v_{n p}=\pi^{-1} \log n+$ $O\left\{(\log n)^{1 / 2}\right\}$.
\end{abstract}

Keywords and phrases. Random polynomials, real zeros, hyperbolic polynomials, Kac-Rice formula.

2000 Mathematics Subject Classification. Primary 60G99.

1. Introduction. Let $n$ and $p$ be integers such that $n \geq 2$ and $p \geq 0$. We suppose that $a_{k}(k=1,2, \ldots, n)$ are independent, normally distributed random variables, each with mean 0 and variance 1 , and we define the random hyperbolic polynomial $f(x)$ so that

$$
f(x)=\sum_{k=1}^{n} k^{p} a_{k} \cosh k x .
$$

We prove the following result.

THEOREM 1.1. Let $v_{n p}$ be the mean number of real zeros of $f(x)$. Then

$$
v_{n p}=\pi^{-1} \log n+O\left\{(\log n)^{1 / 2}\right\} .
$$

The case when $p=0$ was considered by Das [3], whose result was reported by Bharucha-Reid and Sambandham [1, page 110] in the form $v_{n o} \sim \pi^{-1} \log n$. The case when $p=1$ was discussed by Farahmand and Jahangiri [5], who found the result (1.2) in that case.

The principal term in (1.2) is independent of $p$. That behavior does not occur in the algebraic case [4] (replace $\cosh k x$ in (1.1) by $x^{k}$ and let $k$ range from 0 to $n$ ), for which $v_{n p} \sim \pi^{-1}\left\{1+(2 p+1)^{1 / 2}\right\} \log n$ (even if $p$ is a nonnegative real number), and also does not occur in the trigonometric case [2] (replace $\cosh k x$ in (1.1) by $\cos k x$ and count zeros on $(0,2 \pi))$, for which $v_{n p}=\{(2 p+1) /(2 p+3)\}^{1 / 2}(2 n+1)+O\left(n^{1 / 2}\right)$ (even if $p$ is a nonnegative real number). The error term in this last case can be replaced by $O(1)$ when $2 p$ is a nonnegative integer $[6,7,8,9]$.

2. Preliminary analysis. If we apply the Kac-Rice formula to our problem, we see that

$$
v_{n p}=\pi^{-1} \int_{-\infty}^{\infty} F_{n p}(x) d x=2 \pi^{-1} \int_{0}^{\infty} F_{n p}(x) d x
$$


in which

$$
\begin{aligned}
& F_{n p}(x)=\frac{\left\{A_{n p}(x) C_{n p}(x)-B_{n p}^{2}(x)\right\}^{1 / 2}}{A_{n p}(x)}, \\
& A_{n p}(x)=\sum_{k=1}^{n} k^{2 p} \cosh ^{2} k x, \\
& B_{n p}(x)=\sum_{k=1}^{n} k^{2 p+1} \sinh k x \cosh k x, \\
& C_{n p}(x)=\sum_{k=1}^{n} k^{2 p+2} \sinh ^{2} k x .
\end{aligned}
$$

We furnish explicit formulae for the sums in (2.3), (2.4), and (2.5) in the following lemma.

LEMMA 2.1. It is true that

$2^{2 p+2} A_{n p}(x)=(2 n+1)^{2 p} \operatorname{csch} x \sinh z$

$$
\times\left[\sum_{r=0}^{2 p} 2 p C_{r}(2 n+1)^{-r} \varphi_{r}(x)+(2 n+1)^{-2 p}\left(2^{2 p+2} S_{n p}-\delta_{o p}\right) \sinh x \operatorname{csch} z\right],
$$

$2^{2 p+3} B_{n p}(x)=(2 n+1)^{2 p+1} \operatorname{csch} x \sinh z \sum_{r=0}^{2 p+1} 2 p+1 C_{r}(2 n+1)^{-r} \psi_{r}(x)$,

$2^{2 p+4} C_{n p}(x)=(2 n+1)^{2 p+2} \operatorname{csch} x \sinh z$

$$
\times\left[\sum_{r=0}^{2 p+2} 2 p+2 C_{r}(2 n+1)^{-r} \varphi_{r}(x)-(2 n+1)^{-2 p-2} 2^{2 p+4} S_{n, p+1} \sinh x \operatorname{csch} z\right],
$$

in which

$$
\begin{gathered}
z=(2 n+1) x, \\
\varphi_{2 r}(x)=g_{2 r}(x), \quad \varphi_{2 r+1}(x)=g_{2 r+1}(x) \operatorname{coth} z, \\
\psi_{2 r}(x)=g_{2 r}(x) \operatorname{coth} z, \quad \psi_{2 r+1}(x)=g_{2 r+1}(x), \\
g_{r}(x)=\sinh x\left\{\frac{d^{r}(\operatorname{csch} x)}{d x^{r}}\right\}, \\
2 S_{n p}=\sum_{k=1}^{n} k^{2 p},
\end{gathered}
$$

where ${ }_{p} C_{r}$ is the binomial coefficient $p ! /\{r !(p-r) !\}$, and $\delta_{o p}$ is the Kronecker delta, i.e., $\delta_{o p}=1$ when $p=0$ and $\delta_{o p}=0$ when $p \neq 0$. 
With the help of (2.13), the identity $2 \cosh ^{2} k x=\cosh 2 k x+1$, it is clear that

$$
\begin{aligned}
2^{2 p+2} A_{n p}(x) & =\frac{2 d^{2 p}\left\{\sum_{k=1}^{n}(\cosh 2 k x+1)\right\}}{d x^{2 p}}-2 n \delta_{o p}+2^{2 p+2} S_{n p} \\
& =d^{2 p} \frac{\left\{4 A_{n o}(x)\right\}}{d x^{2 p}}-2 n \delta_{o p}+2^{2 p+2} S_{n p} .
\end{aligned}
$$

It is known from [6, equation 2.15] that $4 A_{n o}(x)=2 n-1+\operatorname{csch} x \sinh z$, if $z$ is defined by (2.9). Hence,

$$
2^{2 p+2} A_{n p}(x)=\sum_{r=0}^{2 p} 2 p C_{r}\left\{\frac{d^{r}(\operatorname{csch} x)}{d x^{r}}\right\}\left\{\frac{d^{2 p-r}(\sinh z)}{d x^{2 p-r}}\right\}-\delta_{o p}+2^{2 p+2} S_{n p} .
$$

If the derivatives of $\sinh z$ are calculated and the definitions (2.10) and (2.12) are used, we see that (2.6) is true. In a similar manner, it follows from (2.3), (2.4), and (2.11) that

$$
\begin{aligned}
2^{2 p+3} B_{n p}(x) & =\frac{d\left\{2^{2 p+2} A_{n p}(x)\right\}}{d x}=\frac{d^{2 p+1}(\operatorname{csch} x \sinh z)}{d x^{2 p+1}} \\
& =(2 n+1)^{2 p+1}(\operatorname{csch} x \sinh z) \sum_{r=0}^{2 p+1} 2 p+1 C_{r}(2 n+1)^{-r} \psi_{r}(x),
\end{aligned}
$$

so that (2.7) is true. Finally, (2.8) is a consequence of (2.6) and the identity $C_{n p}(x)=$ $A_{n, p+1}(x)-2 S_{n, p+1}$.

A straightforward calculation, based on (2.6), (2.7), and (2.8), suffices to prove the following lemma.

LEMMA 2.2. It is true that

$$
\begin{aligned}
2^{4 p+6} & \left\{A_{n p}(x) C_{n p}(x)-B_{n p}^{2}(x)\right\}=(2 n+1)^{4 p+2} \operatorname{csch}^{2} x \sinh ^{2} z \\
\times & {\left[\sum_{r=0}^{4 p+2}(2 n+1)^{-r} \theta_{r p}(x)+\Theta_{n p}(x) \sinh x \operatorname{csch} z-\Psi_{n p}(x) \sinh ^{2}(x) \operatorname{csch}^{2} z\right] }
\end{aligned}
$$

in which

$$
\begin{aligned}
\theta_{r p}(x)= & \sum_{s=0}^{r}\left\{2 p C_{s} 2 p+2 C_{r-s} \varphi_{s}(x) \varphi_{r-s}(x)-2 p+1\right. \\
\Theta_{n p}(x)= & \left.(2 n+1)^{-2 p}\left(2^{2 p+2} S_{n p+1}-\delta_{o p-s}\right) \sum_{r=0}^{2 p+2} 2 p+2{ }^{2 p+2} C_{r}(2 n+1)^{-r} \varphi_{r}(x)\right\} \\
& -(2 n+1)^{-2 p-2} 2^{2 p+4} S_{n, p+1} \sum_{r=0}^{2 p} 2 p C_{r}(2 n+1)^{-r} \varphi_{r}(x) \\
\Psi_{n p}(x)= & (2 n+1)^{-4 p-2}\left(2^{2 p+2} S_{n p}-\delta_{o p}\right) 2^{2 p+4} S_{n, p+1}
\end{aligned}
$$

We need the more explicit formulae for $g_{r}(x)$ contained in the following lemma. 
LEMMA 2.3. There are constants $\beta_{r s}(s=0,1, \ldots,[r / 2])$ such that

$$
\begin{aligned}
g_{2 r}(x) & =\sum_{s=0}^{r} \beta_{2 r, s} \operatorname{csch}^{2 s} x, \\
g_{2 r+1}(x) & =\sum_{s=0}^{r} \beta_{2 r+1, s} \operatorname{csch}^{2 s} x \operatorname{coth} x .
\end{aligned}
$$

It follows from (2.12) that (2.21) is true when $r=0$ if $\beta_{00}=1$. A differentiation of (2.12) shows that

$$
g_{r+1}(x)=\frac{d g_{r}}{d x}-g_{r}(x) \operatorname{coth} x .
$$

If (2.21) is true for $r$, we infer from (2.23) that (2.22) is true for $r$, provided that

$$
\beta_{2 r+1, s}=-(2 s+1) \beta_{2 r, s} .
$$

Similarly, the truth of (2.21) with $r$ replaced by $r+1$ is assured when

$$
\beta_{2 r+2, s}=-(2 s+1) \beta_{2 r+1, s}-2 s \beta_{2 r+1, s-1} .
$$

We record for future reference the cases when $r=0,1$, and 2:

$$
g_{0}(x)=1, \quad g_{1}(x)=-\operatorname{coth} x, \quad g_{2}(x)=1+2 \operatorname{csch}^{2} x .
$$

3. Estimates of the terms in (2.6) and (2.17) when $x$ is not too small. We suppose that $x \geq \varepsilon$, in which

$$
\varepsilon=\frac{w}{(2 n+1)}, \quad w=(\log n)^{1 / 2} .
$$

LEMMA 3.1. If $n_{o}=8104$ and $n \geq n_{0}$, the functions $\sinh ^{3} x \operatorname{csch} z$, $\sinh x \operatorname{csch} z$, and $\sinh ^{4} x \operatorname{csch}^{2} z$ are decreasing functions of $x$ when $x \geq \varepsilon$.

We observe that

$$
\begin{aligned}
\frac{\operatorname{csch}^{2} x \operatorname{sech} x \sinh ^{2} z \operatorname{sech} z d\left(\sinh ^{3} x \operatorname{csch} z\right)}{d x} & =3 \tanh z-(2 n+1) \tanh x \\
& <3-(2 n+1) \tanh \varepsilon .
\end{aligned}
$$

Also,

$$
\frac{\cosh ^{2} \varepsilon d\{(2 n+1) \tanh \varepsilon\}}{d n}=\sinh 2 \varepsilon-2 \varepsilon+(2 n w)^{-1}>0 .
$$

Therefore, $(2 n+1) \tanh \varepsilon>3$ when $n \geq n_{0}$ because $(2 n+1) \tanh \varepsilon>3$ when $n=8104$. It follows that $\sinh ^{3} x \operatorname{csch} z$ is decreasing when $x \geq \varepsilon$ and $n \geq n_{0}$. The other functions in the lemma are decreasing because $\left(\sinh ^{3} x \operatorname{csch} z\right)^{1 / 3} \operatorname{csch}^{2 / 3} z$ and $\left(\sinh ^{3} x \operatorname{csch} z\right)^{4 / 3}$ $\operatorname{csch}^{2 / 3} z$ are. The third term on the right hand side of (2.17) is estimated in the following lemma. 
LEMMA 3.2. When $n \geq n_{0}$ and $x \geq \varepsilon$, it is true that

$$
\Psi_{n p}(x) \sinh ^{2} x \operatorname{csch}^{2} z=O\left(w^{4} e^{-2 w}\right)(2 n+1)^{-2} \operatorname{csch}^{2} x .
$$

It follows from an explicit formula [6, equation (2.12)] for $S_{n p}$ that $S_{n p}=O\{(2 n+$ $1)^{2 p+1}$. Then (2.20) and Lemma 3.1 imply that

$$
\begin{aligned}
\Psi_{n p}(x) \sinh ^{4} x \operatorname{csch}^{2} z & =O\left\{(2 n+1)^{2} \sinh ^{4} \varepsilon \operatorname{csch}^{2} w\right\} \\
& =O\left\{(2 n+1)^{2} \varepsilon^{4} \mathrm{e}^{-2 w}\right\} .
\end{aligned}
$$

Lemma 3.2 is an immediate consequence of this result and (3.1).

LEMMA 3.3. When $x \geq \varepsilon$, it is true that $g_{r}(x)=O\left(\varepsilon^{-r}\right), \varphi_{r}(x)=O\left(\varepsilon^{-r}\right)$, and $\psi_{r}(x)=O\left(\varepsilon^{-r}\right)$.

The lemma follows immediately from (2.10), (2.11), (2.21), and (2.22), and the facts that

$$
\begin{aligned}
& \operatorname{csch} x \leq \operatorname{csch} \varepsilon<\varepsilon^{-1}, \\
& \operatorname{coth} x \leq \operatorname{coth} \varepsilon<\varepsilon^{-1} \cosh \varepsilon_{o}, \\
& \operatorname{coth} z \leq \operatorname{coth} w \leq \operatorname{coth} w_{0},
\end{aligned}
$$

in which $\varepsilon_{o}=w_{0} /\left(2 n_{o}+1\right)$ and $w_{0}=\left(\log n_{0}\right)^{1 / 2}$. Now, we can estimate the second term on the right-hand side of (2.17).

LEMMA 3.4. When $n \geq n_{0}$ and $x \geq \varepsilon$, it is true that

$$
\Theta_{n p}(x) \sinh x \operatorname{csch} z=O\left(w^{3} \mathrm{e}^{-w}\right)(2 n+1)^{-2} \operatorname{csch}^{2} x .
$$

We deduce from (2.19), Lemmas 3.1 and 3.3, and the earlier observation that $S_{n p}=$ $O\left\{(2 n+1)^{2 p+1}\right\}$ that

$$
\begin{aligned}
\Theta_{n p}(x) \sinh ^{3} x \operatorname{csch} z & =O\left\{(2 n+1) \sum_{r=0}^{2 p+2} O\left(w^{-r}\right) \sinh ^{3} \varepsilon \operatorname{csch} w\right\} \\
& =O\left\{(2 n+1) \varepsilon^{3} \mathrm{e}^{-w}\right\}=O\left(w^{3} \mathrm{e}^{-w}\right)(2 n+1)^{-2} .
\end{aligned}
$$

This equation suffices to prove Lemma 3.4.

The analysis to obtain an estimate for $\theta_{r p}$ is more recondite. We use (2.10), (2.11), (2.18), and the identity $\operatorname{coth}^{2} z=1+\operatorname{csch}^{2} z$, to see that

$$
\theta_{2 r, p}=\sum_{s=0}^{2 r} L_{2 r, s p} g_{s}(x) g_{2 r-s}(x)+M_{r p}(x) \operatorname{csch}^{2} z,
$$

in which

$$
\begin{aligned}
L_{r s p}= & { }_{2 p} C_{s} 2 p+2 C_{r-s}-2 p+1 \\
M_{r p}(x)= & \sum_{s=0}^{r-1} 2 p C_{2 s+1} 2 p+2 C_{2 r-2 s-1} C_{2 s+1}(x) g_{2 r-2 s-1}(x) \\
& -\sum_{s=0}^{r} 2 p+1 C_{2 s} 2 p+1 \\
C_{2 r-2 s} & g_{2 s}(x) g_{2 r-2 s}(x) .
\end{aligned}
$$


In a similar manner, we also see that

$$
\theta_{2 r+1, p}(x)=\sum_{s=0}^{2 r+1} L_{2 r+1, s p} g_{s}(x) g_{2 r+1-s}(x) \operatorname{coth} z .
$$

Because we infer from (3.9) and Lemma 3.3 that $M_{r p}(x)=O\left(\varepsilon^{-2 r}\right)$, it follows, from (3.9) and (3.12), that

$$
\theta_{r p}(x)=\sum_{s=0}^{r} L_{r s p} g_{s}(x) g_{r-s}(x)(\operatorname{coth} z)^{u_{r}}+O\left(\varepsilon^{-2 r}\right) \operatorname{csch}^{2} z
$$

in which $u_{r}=\left\{1-(-1)^{r}\right\} / 2$. Moreover, Lemma 2.3 implies that

$$
g_{r}(x)=\sum_{h=0}^{[r / 2]} \beta_{r h} \operatorname{csch}^{2 h} x(\operatorname{coth} z)^{u_{r}},
$$

so that there are constants $\gamma_{r s h}$ such that

$$
g_{s}(x) g_{r-s}(x)=\sum_{h=0}^{[r / 2]} \gamma_{r s h} \operatorname{csch}^{2 h} x(\operatorname{coth} x)^{u_{r}} .
$$

In the derivation of (3.15), it is helpful to consider separately the cases when $r$ is even and $r$ is odd. When $r$ is even and $s$ is odd, we also need the identity $\operatorname{coth}^{2} x=1+$ $\operatorname{csch}^{2} x$. An easy induction using (2.24) and (2.25) when $s=0$ shows that $\beta_{\text {ro }}=(-1)^{r}$; hence $\gamma_{r s o}=(-1)^{r}$.

The combinatorial identity

$$
\sum_{s=0}^{r} L_{r s p}=0
$$

is well known (and is easy to prove). We now deduce, from (3.13), (3.15), and (3.16), that

$$
\begin{aligned}
\theta_{r p}(x) \sinh ^{2} x= & \sum_{s=0}^{r} L_{r s p} \sum_{h=1}^{[r / 2]} \gamma_{r s h} \operatorname{csch}^{2 h-2} x(\operatorname{coth} x \operatorname{coth} z)^{u_{r}} \\
& +O\left(\varepsilon^{-r}\right) \sinh ^{2} x \operatorname{csch}^{2} z .
\end{aligned}
$$

We showed in the proof of Lemma 3.3 that

$$
\operatorname{csch} x=O\left(\varepsilon^{-1}\right), \quad \operatorname{coth} x=O\left(\varepsilon^{-1}\right), \quad \operatorname{coth} z=O(1) .
$$

Because it follows from Lemma 2.3 that

$$
\sinh ^{2} x \operatorname{csch}^{2} z \leq \sinh ^{2} \varepsilon \operatorname{csch}^{2} w=O\left(\varepsilon^{2} \mathrm{e}^{-2 w}\right),
$$

we conclude that the following lemma is true.

LEMMA 3.5. When $n \geq n_{o}$ and $x \geq \varepsilon$, it is true that

$$
\theta_{r p}(x)=O\left(\varepsilon^{2-r}\right)\left\{1+O\left(\mathrm{e}^{-2 w}\right)\right\} \operatorname{csch}^{2} x .
$$


We also need the more precise estimates of $\theta_{r p}(x)$ when $r=0,1$, and 2, deducible from (2.10), (2.11), (2.18), and (2.26), that are recorded below:

$$
\begin{aligned}
\theta_{0 p}(x) & =-\operatorname{csch}^{2} z=O\left(w^{2} \mathrm{e}^{-2 w}\right)(2 n+1)^{-2} \operatorname{csch}^{2} x \\
\theta_{1 p}(x) & =0 \\
\theta_{2 p}(x) & =\left(1-4 p^{2} \operatorname{csch}^{2} z+2 p \sinh x \operatorname{csch} z\right) \operatorname{csch}^{2} x \\
& =\left\{1+O\left(\mathrm{e}^{-2 w}\right)+(2 n+1)^{-1} O\left(w \mathrm{e}^{-w}\right)\right\} \operatorname{csch}^{2} x \\
& =\left\{1+O\left(\mathrm{e}^{-2 w}\right)\right\} \operatorname{csch}^{2} x .
\end{aligned}
$$

Finally, the methods used above can be applied to (2.6) to yield an easy proof of the following lemma.

LEMMA 3.6. When $n \geq n_{o}$ and $x \geq \varepsilon$, it is true that

$$
2^{2 p+2} A_{n p}=(2 n+1)^{2 p} \operatorname{csch} x \sinh z\left[1+O\left(w^{-1}\right)\right] .
$$

Proof OF THeOrem 1.1. If we use Lemmas 3.2, 3.4, and 3.5, we infer from (2.17), and (3.21) that, when $n \geq n_{o}$ and $x \geq \varepsilon$,

$$
2^{4 p+6}\left\{A_{n p}(x) C_{n p}(x)-B_{n p}^{2}(x)\right\}=(2 n+1)^{4 p} \operatorname{csch}^{4} x \sinh ^{2} z\left[1+O\left(w^{-1}\right)\right] .
$$

It now follows from (2.2) and Lemma 3.6 that, when $n \geq n_{0}$ and $x \geq \varepsilon$,

$$
\begin{aligned}
2 F_{n p}(x) d x & =\left\{1+O\left(w^{-1}\right)\right\} \operatorname{csch} x, \\
2 \int_{\varepsilon}^{\infty} F_{n p}(x) d x & =\left\{1+O\left(w^{-1}\right)\right\} \log \left\{\operatorname{coth}\left(\frac{\varepsilon}{2}\right)\right\} \\
& =\left\{1+O\left(w^{-1}\right)\right\}\left\{1+O\left(w^{-2} \log w\right)\right\} \log n, \\
2 \pi^{-1} \int_{\varepsilon}^{\infty} F_{n p}(x) d x & =\pi^{-1} \log n+O\left\{(\log n)^{1 / 2}\right\} .
\end{aligned}
$$

Next, we observe that (2.2), (2.3), and (2.5) imply that

$$
\begin{gathered}
0 \leq C_{n p}(x) \leq n^{2} \sum_{k=1}^{n} k^{2 p} \sinh ^{2} k x<n^{2} A_{n p}(x), \\
0 \leq F_{n p}(x) \leq\left\{\frac{C_{n p}(x)}{A_{n p}(x)}\right\}^{1 / 2}<n, \\
2 \pi^{-1} \int_{0}^{\varepsilon} F_{n p}(x) d x<2 \pi^{-1} n \varepsilon<\pi^{-1} w=O\left\{(\log n)^{1 / 2}\right\} .
\end{gathered}
$$

If we add (3.26) and (3.29) and use (2.1), we see that the theorem is true.

\section{REFERENCES}

[1] A. T. Bharucha-Reid and M. Sambandham, Random Polynomials, Probability and Mathematical Statistics, Academic Press, Inc., Orlando, 1986. MR 87m:60118. Zbl 615.60058.

[2] M. Das, The average number of real zeros of a random trigonometric polynomial, Math. Proc. Cambridge Philos. Soc. 64 (1968), 721-729. MR 38\#1720. Zbl 169.48902. 
[3] _ On the real zeros of a random polynomial with hyperbolic elements, Ph.D. thesis, Utkal University, India, 1971.

[4] _ _ Real zeros of a class of random algebraic polynomials, J. Indian Math. Soc. (N.S.) 36 (1972), 53-63. MR 48 1318. Zbl 293.60058.

[5] K. Farahmand and M. Jahangiri, On real zeros of random polynomials with hyperbolic elements, Internat. J. Math. Math. Sci. 21 (1998), no. 2, 347-350. MR 99i:60107. Zbl 908.60042.

[6] J. E. Wilkins, Jr., Mean number of real zeros of a random trigonometric polynomial, Proc. Amer. Math. Soc. 111 (1991), no. 3, 851-863. MR 91f:60093. Zbl 722.60047.

[7] _ Mean number of real zeros of a random trigonometric polynomial. II, Topics in polynomials of one and several variables and their applications (River Edge, NJ) (Th. M. Rassias, H. M. Srivastava, and A. Yanushauskas, eds.), World Sci. Publishing, 1993. MR 95g:60067. Zbl 857.60047.

[8]___ Mean number of real zeros of a random trigonometric polynomial. IV, J. Appl. Math. Stochastic Anal. 10 (1997), no. 1, 67-70. MR 98e:60079. Zbl 880.60057.

[9] J. E. Wilkins, Jr. and S. A. Souter, Mean number of real zeros of a random trigonometric polynomial. III, J. Appl. Math. Stochastic Anal. 8 (1995), no. 3, 299-317. MR 96j:60095. Zbl 828.60035.

Wilkins: DePARTMENT of Mathematics, Clark Atlanta University, ATLANTA, GA 30314, USA 


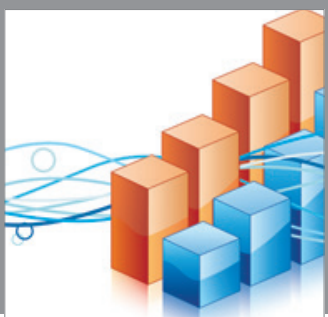

Advances in

Operations Research

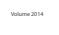

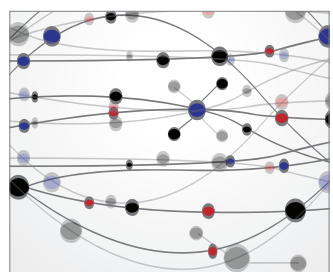

\section{The Scientific} World Journal
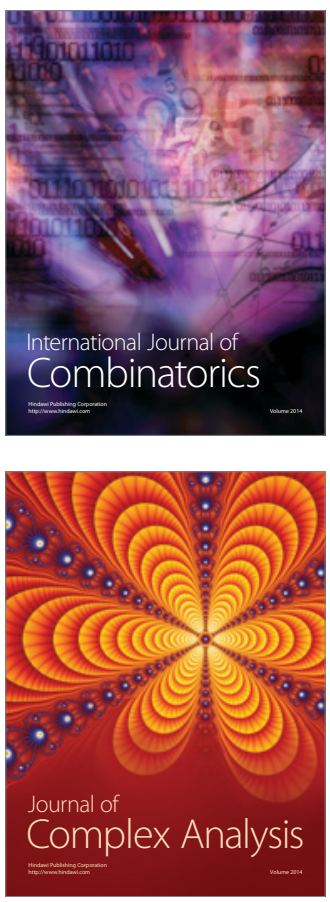

International Journal of

Mathematics and

Mathematical

Sciences
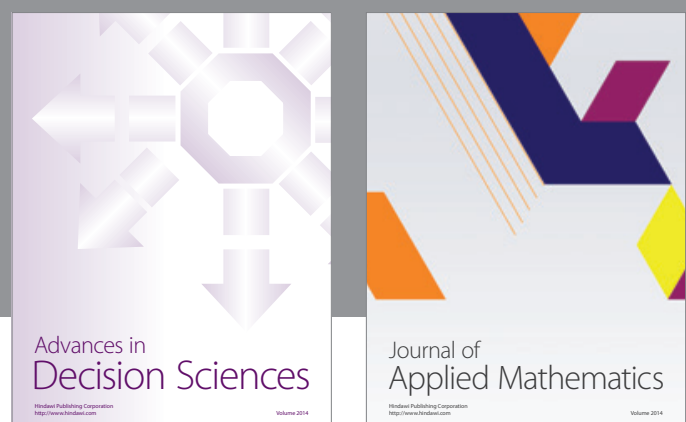

Journal of

Applied Mathematics
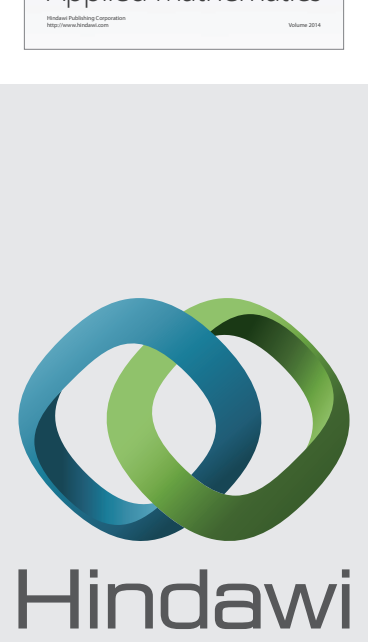

Submit your manuscripts at http://www.hindawi.com
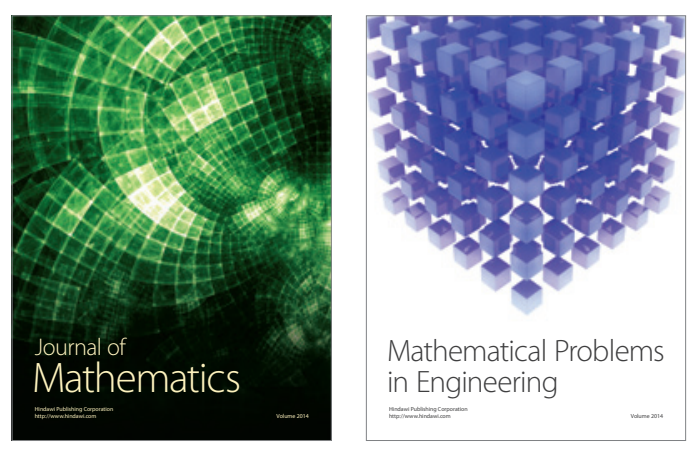

Mathematical Problems in Engineering
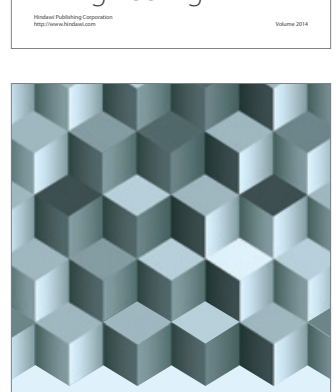

Journal of

Function Spaces
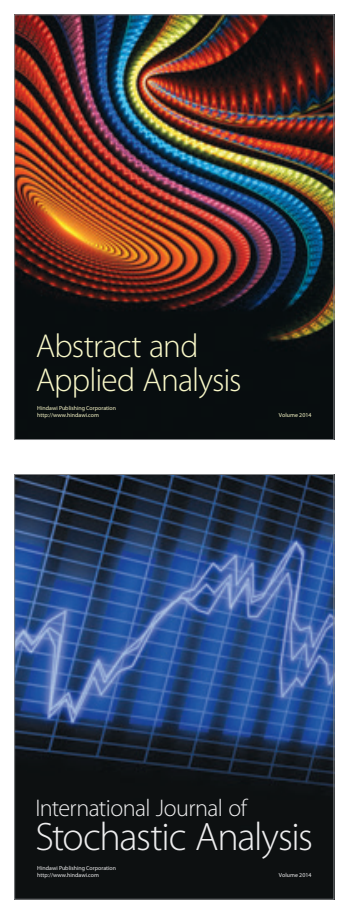

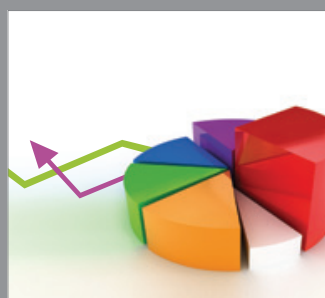

ournal of

Probability and Statistics

Promensencen
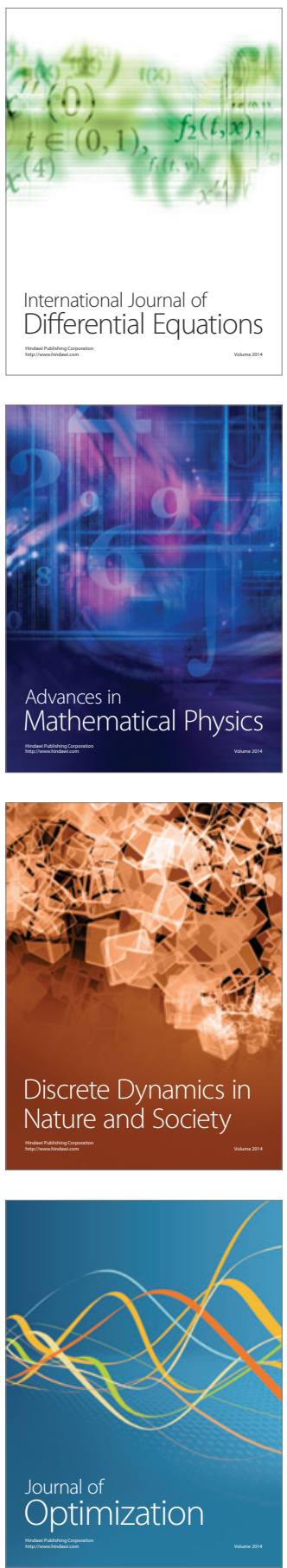